\title{
Transmissible Gastroenteritis Virus Papain-Like Protease 1 Antagonizes Production of Interferon- $\beta$ through Its Deubiquitinase Activity
}

\author{
Xiaoliang Hu, ${ }^{1}$ Jin Tian, ${ }^{1}$ Hongtao Kang, ${ }^{1}$ Dongchun Guo, ${ }^{1}$ Jiasen Liu, ${ }^{1}$ Dafei Liu, ${ }^{1}$ \\ Qian Jiang, ${ }^{1}$ Zhijie Li, ${ }^{1}$ Juanjuan $\mathbf{Q u},{ }^{2}$ and Liandong $\mathbf{Q u}{ }^{1}$ \\ ${ }^{1}$ State Key Laboratory of Veterinary Biotechnology, Harbin Veterinary Research Institute, Chinese Academy of Agricultural Sciences, \\ Harbin 150001, China \\ ${ }^{2}$ College of Life Science, Northeast Agricultural University, Harbin, China
}

Correspondence should be addressed to Liandong Qu; qld@hvri.ac.cn

Received 23 May 2017; Revised 14 August 2017; Accepted 24 August 2017; Published 23 October 2017

Academic Editor: Yanjin Zhang

Copyright $\odot 2017$ Xiaoliang Hu et al. This is an open access article distributed under the Creative Commons Attribution License, which permits unrestricted use, distribution, and reproduction in any medium, provided the original work is properly cited.

Coronaviruses (CoVs), such as human coronavirus NL63 (HCoV-NL63), severe acute respiratory syndrome CoV (SARS-CoV), murine hepatitis virus (MHV), porcine epidemic diarrhea virus (PEDV), and Middle East Respiratory Syndrome Coronavirus (MERS-CoV), encode papain-like (PL) proteases that inhibit Sendai virus- (SeV-) induced interferon (IFN- $\beta$ ) production. Recently, the crystal structure of transmissible gastroenteritis virus (TGEV) PL1 has been solved, which was similar to that of SARS-CoV PL2 ${ }^{\text {pro }}$, which may antagonize host innate immunity. However, very little is known about whether TGEV PL1 can antagonize host innate immune response. Here, we presented evidence that TGEV PL1 encoded by the replicase gene could suppress the IFN- $\beta$ expression and inhibit the nuclear translocation of interferon regulatory factor 3 (IRF3). The ability to antagonize IFN- $\beta$ production was dependent on the intact catalytic activity of PL1. Furthermore, TGEV PL1 exerted deubiquitinase (DUB) activity which strongly inhibited the retinoic acid-induced gene I- (RIG-1-) and stimulator of interferon gene- (STING-) dependent IFN expression. Our data collectively suggest that TGEV PL1 can inhibit the IFN- $\beta$ expression and interfere with RIG-1- and STING-mediated signaling through a viral DUB activity. Our study has yielded strong evidence for the TGEV PL1 mechanisms that counteract the host innate immunity.

\section{Introduction}

The innate immune system is the first line of defense that protects the host against viral infection, and the induction of IFN $-\alpha / \beta$ is a crucial antiviral mechanism of the innate immune system [1]. The initiation of IFN expression is triggered by pathogen-associated molecular patterns (PAMPs) through host pattern recognition receptors (PRRs) [2]. After viral RNAs are sensed by PRRs, signals are transmitted to different downstream adaptor molecules (such as IFN- $\beta$ promoter stimulator 1 (IPS-1)); and then I $\kappa$ B kinase- (IKK-) related kinases are recruited. Next, interferon regulatory factor 3 (IRF3), nuclear factor $\kappa \mathrm{B}$ (NF- $\kappa \mathrm{B})$, and ATF-2/c-jun are activated by the kinase complexes and translocate to the nucleus and directly induce the expression of type I IFNs [3].
TGEV is an enveloped virus belonging to the Coronaviridae $(\mathrm{CoV})$ family and the Nidovirales order. CoVs are positive-strand RNA viruses that replicate in the cytoplasm of infected cells [4]. CoVs encode two types of cysteine proteases, $\mathrm{M}^{\text {pro }}$, and papain-like proteases, PL1 and PL2, which contained nonstructural protein 5 (nsp5) and nsp3, respectively. $\mathrm{PL}^{\mathrm{pro}}$ is served mainly as in processing of the replicase ppla and pplab polypeptides [5]. Other than their role in replicase polyprotein processing, PL2 domains possess an additional but related enzymatic activity, in HCoV-NL63 [6], MHV [7], SARS-CoV [8, 9], and MERS$\mathrm{CoV}[10]$, through their deubiquitination (DUB) enzymes, which play a key role in antagonizing IFN induction. However, TGEV PL1 processes the nsp $2 / \mathrm{nsp} 3$ site and is capable of hydrolyzing isopeptide bonds in both Lys48- and 
Lys63-linked polyubiquitin chains [11]. Whether TGEV PL1 could antagonize the production of IFNs was unknown.

In the present study, we found that TGEV PL1 encoded by the replicase gene could suppress the IFN- $\beta$ expression and inhibit the nuclear translocation of interferon regulatory factor 3 (IRF3) and exerted deubiquitinase (DUB) activity which strongly inhibited the retinoic acid-induced gene I- (RIG-1-) and stimulator of interferon gene- (STING-) dependent IFN expression.

\section{Methods}

Cells and Viruses. HEK293T cells and PK-15 cells were cultured in Dulbecco's modified Eagle's medium (Hyclone, Logan, USA) containing $10 \%(\mathrm{v} / \mathrm{v})$ fetal calf serum supplemented with penicillin $\left(100 \mathrm{U} \mathrm{ml}^{-1}\right)$ and streptomycin $\left(100 \mu \mathrm{g} \mathrm{ml}^{-1}\right)$. Sendai virus $(\mathrm{SeV})$ was obtained from the Centre of Virus Resource and Information (Wuhan Institute of Virology, Chinese Academy of Sciences).

Plasmids and Agents. IFN- $\beta$-Luc, 4x PRDIII/I-Luc (referred to as IRF3-Luc), $4 \mathrm{x}$ AP-1-Luc, and $4 \mathrm{x}$ NF- $\kappa \mathrm{B}-$ Luc luciferase reporter plasmids were constructed according to an earlier protocol [12]. Accession numbers of STING, IRF3, and MAVS were KC860780, KC860781, and KC860782, respectively. Expression plasmids for RIG-1 (p-Flag-RIG-1) and TBK-1 (p-Flag-TBK-1) were generated with the following primers: RIG-1 forward, 5 '-TTTGGATCCATGACAGCAGAGCAGCGGCGGAAT-3', RIG-1 reverse $5^{\prime}$-TTTAAGCTTCACTCAAGGTTCGGGATTCCCTG-3'; TBK-1 forward, $5^{\prime}$-TTTGAATTCATGCAGAGCACTTCTAATCATCTTT-3', TBK-1 reverse $5^{\prime}$-TTTAGATCTTAAAGACAGTCAACATTGCGAA-3'. To construct the DNA expression vector, pMyc-PL1, pFlag-PL1, and pPL1-Myc, encoding TGEV PL1, standard reverse transcription- (RT-) PCR was applied to amplify cDNA of the total RNA extracted from PK-15 cells infected with the TGEV strain HX, using the following primers: PL1-forward, 5'-GTACAAGAAGCTGAACAATTTAA-3' (3498-3520 bp), PL1 reverse, $5^{\prime}$ ATCGTTTTTAGGACTTTGAATTT-3' (4249-4271 bp). All constructs were validated via DNA sequencing. pDsRed2Mito was purchased from Clontech (Tokyo, Japan). Transfection agent was performed with X-tremeGENE HP (Roche, Switzerland) per the manufacturer's instructions.

Luciferase Reporter Gene Assay. HEK-293T cells grown in 24 -well plates were cotransfected with $0.2 \mu \mathrm{g} /$ well reporter plasmid, $0.02 \mu \mathrm{g} /$ well pRL-TK plasmid (Promega, Madison, USA) as an internal control for normalization of transfection efficiency, and the indicated expression or empty control vector plasmid. Where indicated, cells were also mockinfected or infected with $\mathrm{SeV}$ (100 hemagglutinating activity units/well) at $10 \mathrm{~h}$ after cotransfection. Cells were subsequently lysed, and firefly and Renilla luciferase activities were determined with the Dual-Luciferase reporter assay system (Promega, Madison, USA), according to the manufacturer's protocol. Data are presented as mean relative luciferase units \pm standard deviation from triplicate samples. For statistical analysis, data were compared between empty vector- and TGEV PL1-transfected groups with the unpaired, two-tailed Student's $t$-test using SPSS 11.0 software. $P$ values $<0.05$ were considered statistically significant [13].
ELISA. Cell supernatants of transfected PK-15 cells were centrifuged at $3,000 \mathrm{~g}$ for $5 \mathrm{~min}$ to remove cell debris and stored at $-80^{\circ} \mathrm{C}$ until use. Secreted IFN- $\beta$ in the cell supernatants was determined using commercial Porcine IFN- $\beta$ (Interferon Beta) ELISA Kit (Elabscience, China) according to the manufacturer's instructions.

Immunoblot Analysis. HEK293T cells were cultured in 6well plates and $60 \mathrm{~mm}$ dishes were transfected with the appropriate plasmids. After $36 \mathrm{~h}$, cells were harvested by the addition of lysis buffer and protein concentrations in whole cell extracts measured. Equal amounts of samples were subjected to SDS-PAGE and analyzed for TGEV PL1, STING, TBK-1, and IRF3 proteins via immunoblotting using HA, Flag, or GFP-tagged antibodies (Sigma, St Louis, USA). Expression of p-IRF3, IRF3, and GAPDH was detected with the rabbit-anti p-IRF3 (ab76493), IRF3 (ab68481) (Abcam, Cambridge, UK), and a mouse anti-GAPDH monoclonal antibody (Sigma, St Louis, USA).

Assay of Deubiquitinase Activity in Cultured Cells. HEK293T cells were cotransfected with pcDNA3.1-HA-Ub plus the indicated amounts of TGEV PL1, p-Flag-RIG-1, and p-FlagSTING constructs. The effect of TGEV PL1 on ubiquitinated proteins in cultured cells was assessed by immunoblot analysis.

Coimmunoprecipitation Analysis. Coimmunoprecipitation experiments were performed on HEK293T cells transfected with the indicated expression plasmids as described in an earlier report [14].

Immunofluorescence Assay. HEK293T cells were plated on fibronectin-treated glass coverslips in 24-well plates. To evaluate the localization of TGEV PL1, cells were cotransfected with plasmid DNA expressing Flag-PL (500 ng per well) and pDsRed-Mito (500 ng per well) using X-treme GENE HP, according to the manufacturer's protocol. HEK293T cells were cotransfected with IRF3-GFP (500 ng per well) and empty vector (500 ng per well) or IRF3-GFP (500 ng per well) and Flag-PL1 (500 ng per well). $24 \mathrm{~h}$ after transfection, cells were infected with $\mathrm{SeV}$ (100 hemagglutinating activity units/well) for $16 \mathrm{~h}$. Next, cells were fixed with $4 \%$ paraformaldehyde for $30 \mathrm{~min}$ and permeated with $0.1 \%$ Triton X-100 for $15 \mathrm{~min}$ at room temperature. After three washes with PBS, cells were blocked with PBS containing $5 \%$ bovine serum albumin for $2 \mathrm{~h}$, followed by incubation with a mouse monoclonal antibody against Flag (1:100) for $1 \mathrm{~h}$ at room temperature. Cells were treated with fluorescein isothiocyanate-labeled goat anti-mouse (Sigma, St Louis, USA) for $1 \mathrm{~h}$, and subsequently with $4^{\prime}, 6$-diamidino2-phenylindole (DAPI) for $15 \mathrm{~min}$ at room temperature. Samples were washed with PBS, and fluorescent images were acquired under a confocal laser scanning microscope (TCS SP5; Leica, Solms, Germany).

Detection of STING Dimers. To assess the formation of STING dimers, HEK293T cells were transfected with Flag-STING (500 ng per well) and the lysates were subjected to Western blot, as described earlier [15], with the indicated antibodies. 


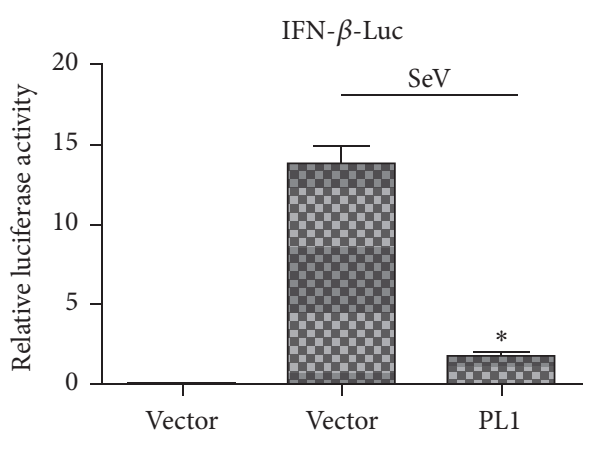

(a)



(c)

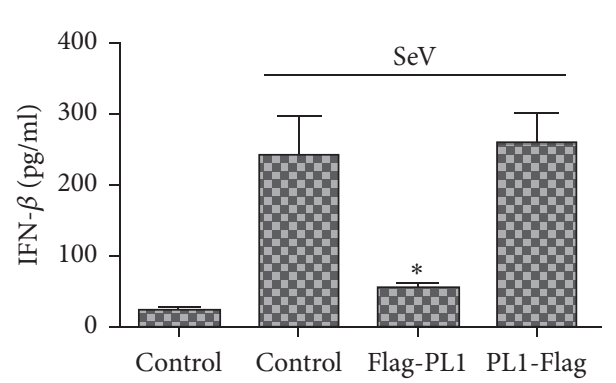

(e)



(b)

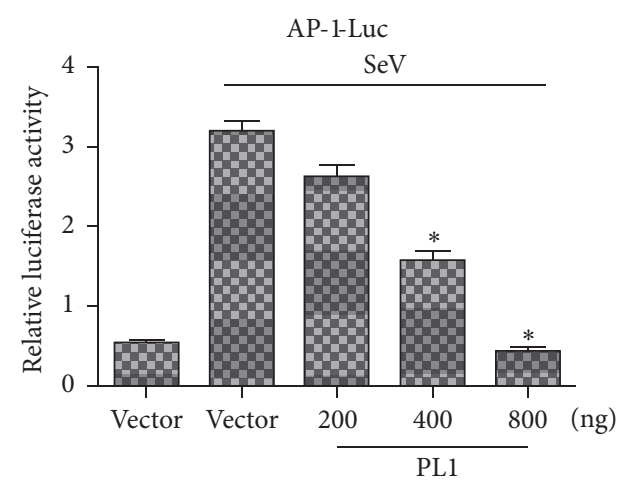

(d)

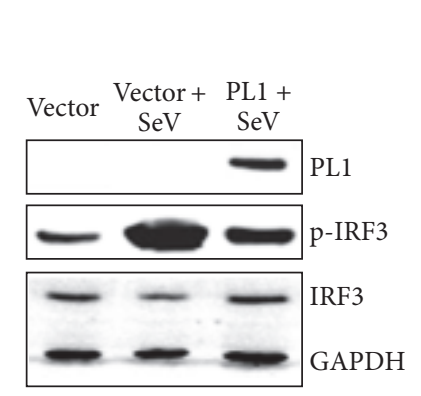

(f)

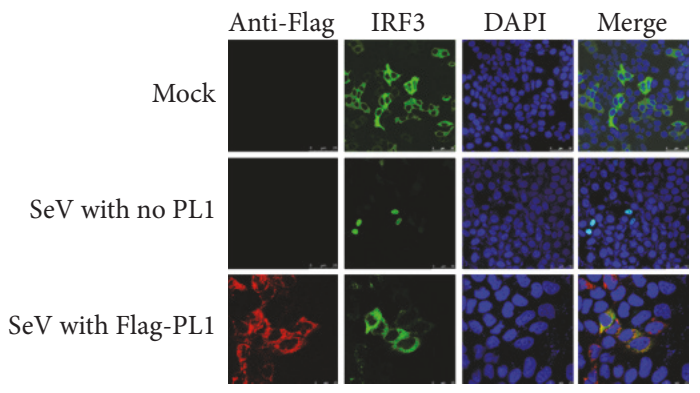

$(\mathrm{g})$

FIGURE 1: TGEV PL1 inhibits SeV-induced expression of IFN- $\beta$-Luc in a dose-dependent manner. (a) HEK-293T cells grown in 24-well plates were transfected with $1 \mu \mathrm{g}$ plasmid encoding Myc-TGEV PL1 or empty vector and infected with SeV $24 \mathrm{~h}$ later (100 hemagglutinating activity units/well). After $10 \mathrm{~h}$ infection, cells were lysed, and activities of IFN- $\beta$-Luc and pRL-TK reporters were determined according to the manufacturer's protocol. Myc-TGEV PL1 inhibits the activities of IRF3 (b), NF- $\kappa$ B (c), and AP-1 (d). Luciferase activities were assayed as described for (a). Results represent the means and standard deviations of data from three independent experiments. (e) PK-15 cells grown in 12-well plates were transfected with $500 \mathrm{ng}$ plasmid Flag-PL1 and empty vector and infected with SeV $24 \mathrm{~h}$ later. After $10 \mathrm{~h}$ infection, cell supernatants were collected and analyzed for IFN- $\beta$ production by ELISA. (f) HEK-293T cells grown in 6-well plates were transfected with $2 \mu \mathrm{g}$ plasmid encoding Myc-TGEV PL1 or empty vector and infected with SeV $24 \mathrm{~h}$ later (100 hemagglutinating activity units/well). After $10 \mathrm{~h}$ infection, cells were lysated and detected by Western blot. (g) Immunofluorescence microscopy of HeLa cells expressing Flag-PL1 and IRF3GFP. Cells were fixed $24 \mathrm{~h}$ after transfection and $10 \mathrm{~h} \mathrm{SeV}$ infection, and Flag-tagged products were visualized using confocal microscopy. Asterisks indicate statistical significance $(P<0.05)$.

\section{Results}

3.1. TGEV PL1 Is an IFN Antagonist. The crystal structure of TGEV PL1 has been determined [11]. The structure of TGEV PL1 is similar to that of SARS-CoV PL2 ${ }^{\text {pro }}$. In order to determine whether TGEV PL1 is capable of blocking IFN- $\beta$ production, we assessed IFN- $\beta$ promoter activity in the presence of PL1 (Figure 1(a)). HEK 293T cells were cotransfected with
TGEV PL1 and IFN- $\beta$ luciferase or Renilla luciferase reporter plasmids for $24 \mathrm{~h}$ and subsequently infected with $\mathrm{SeV}$ to activate the RIG-1-dependent IFN- $\beta$ expression pathway. We observed the inhibition of $\mathrm{SeV}$-induced IFN- $\beta$ promoter activation in the presence of PL1, similar to the antagonistic function of NL63 PLP2 and porcine epidemic diarrhea virus (PEDV) PLP2, clearly indicating that TGEV PL1 could act as an interferon antagonist. 


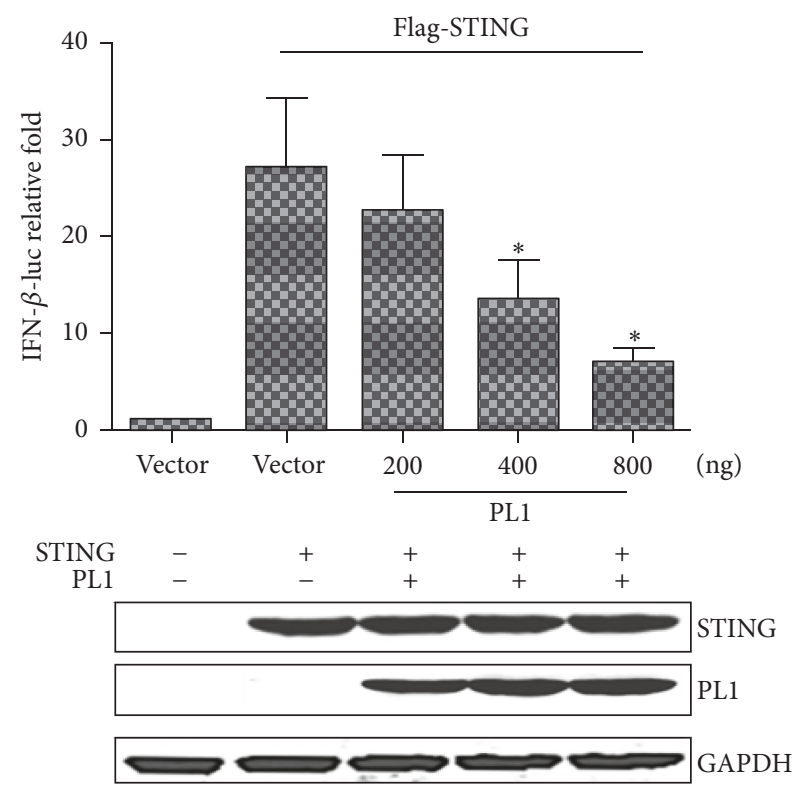

(a)
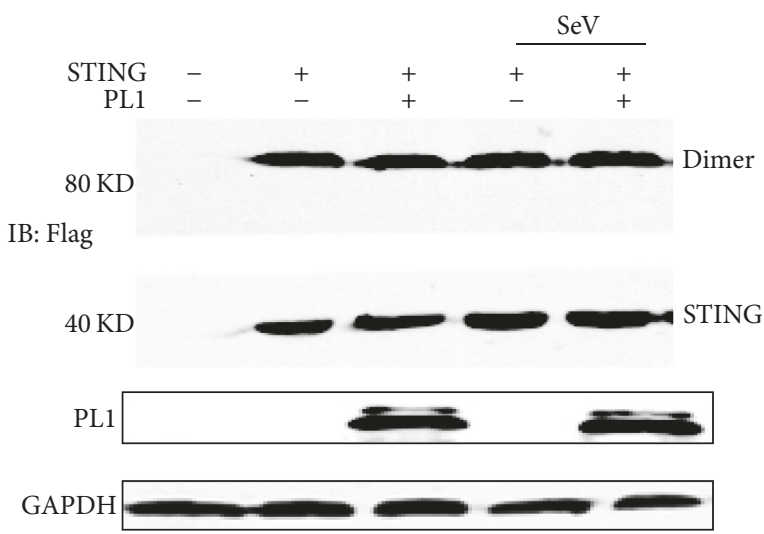

(b)

FIGURE 2: Expression of TGEV PL1 inhibits STING-mediated activation of IFN- $\beta$-Luc in a dose-dependent manner. (a) HEK293T cells were cotransfected with certain Flag-PL1, IFN- $\beta$-Luc, pRL-TK, and $500 \mathrm{ng}$ Flag-STING or $500 \mathrm{ng}$ empty vector. Asterisks indicate statistical significance $(P<0.05)$. Proteins were assayed using Western blot with anti-Flag and GAPDH antibodies. (b) HEK293T cells were cotransfected with $500 \mathrm{ng}$ STING and/or $500 \mathrm{ng}$ TGEV PL1, and/or infected with SeV. Cell lysates were separated via SDS-PAGE and subjected to immunoblotting with the relevant antibodies.

To establish the mechanisms by which PL1 inhibits IFN$\beta$ expression, transcriptional activities of NF- $\kappa \mathrm{B}$, IRF3, and AP-1 were analyzed using the luciferase assay to identify the precise transcription factor involved. Notably, the luciferase activities of all three transcription factors were significantly inhibited by TGEV PL1 in a dose-dependent manner (Figures 1(b), 1(c), and 1(d)). Furthermore, Flag-PL1 also significantly inhibited IFN- $\beta$ production in PK-15 cells at protein level (Figure 1(e)), which was further confirmed with the result that TGEV PLl could block the production of interferon. To further establish whether TGEV PL1 affects IRF3 phosphorylation or migration from the cytoplasm to nucleus, HEK293T cells were transfected with TGEV PL1 and/or IRF3EGFP. Then the result was analyzed using Western blot and confocal microscopy. In Figure 1(f), the level of p-IRF3 was decreased significantly by TGEV PL1 compared with that of $\mathrm{SeV}$-induced. Furthermore, IRF3-EGFP was located in the cytoplasm compared with mock-infected HEK293T cells but translocated to the nucleus when the cells were inoculated with $\mathrm{SeV}$. In contrast, after being inoculated with $\mathrm{SeV}$, it was found that IRF3-EGFP was translocated from cytoplasm to nuclear in mock infected HEK293T cell, which was not observed in cells transfected with TGEV PL1 (Figure 1(g)). Our results collectively suggested that TGEV PL1 suppressed IFN- $\beta$ transcription by interfering with NF- $\kappa$ B-, IRF3-, and AP-1 signaling-mediated IFN expression.

3.2. TGEV PL1 Antagonized STING-Dependent Signaling. To determine whether TGEV PL1 is capable of blocking STINGmediated activation of the IFN- $\beta$ promoter, we assessed promoter activity in the presence of STING along with increasing amounts of TGEV PL1. Stimulation of HEK293T cells with STING alone resulted in a robust increase in IFN$\beta$ promoter activity. Coexpression of STING and TGEV PL1 induced a dose-dependent decrease in IFN- $\beta$ activity, clearly indicating antagonistic activity of TGEV PL1 on STINGmediated activation of the IFN- $\beta$ promoter (Figure $2(\mathrm{a})$ ).

STING dimerization is reduced in the presence of $\mathrm{HCoV}$ NL63. STING dimmers are visualized as an $80 \mathrm{kD}$ band on SDS-PAGE. To further determine whether TGEV PL1 inhibits STING-mediated signaling through disrupting the stability of STING dimers, HEK293T cells were cotransfected with plasmid DNA expressing STING in the presence or absence of TGEV PL1 and SeV, and cell lysates were evaluated for dimmers via immunoblotting (Figure 2(b)). Interestingly, the results indicated that STING dimerization was not affected by TGEV PL1.

3.3. The Catalytic Activity of TGEV PL1 Is Essential for Inhibiting IFN- $\beta$ Expression. To determine whether catalytic activity is required for TGEV PL1-mediated inhibition of IFN- $\beta$ expression, HEK293T cells were cotransfected with alanine mutants of three conserved catalytic residues of TGEV PL1 (C32A, H183A, and D196A) with or without RIG1 , MAVS, STING, or TBK- 1 , and IFN- $\beta$-Luc and pRL-TK plasmids, followed by infection with $\mathrm{SeV}$ to activate IFN- $\beta$ promoter activity. TGEV PL1 mutation at two of the catalytic sites (C32A and H183A) led to almost complete loss of IFN antagonistic activity, relative to wild-type TGEV PL1, but the D196A mutant showed a little inhibition for IFN- $\beta$ promoter 


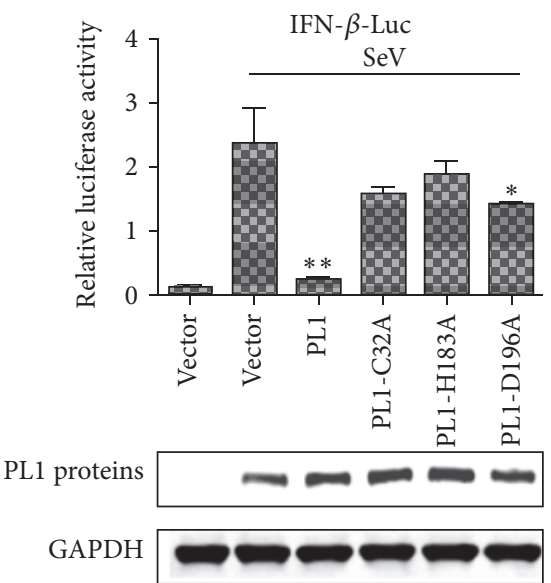

(a)

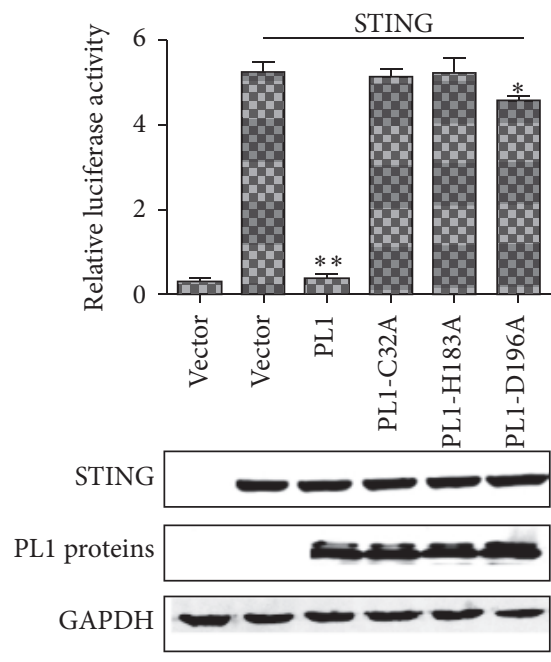

(d)



(b)

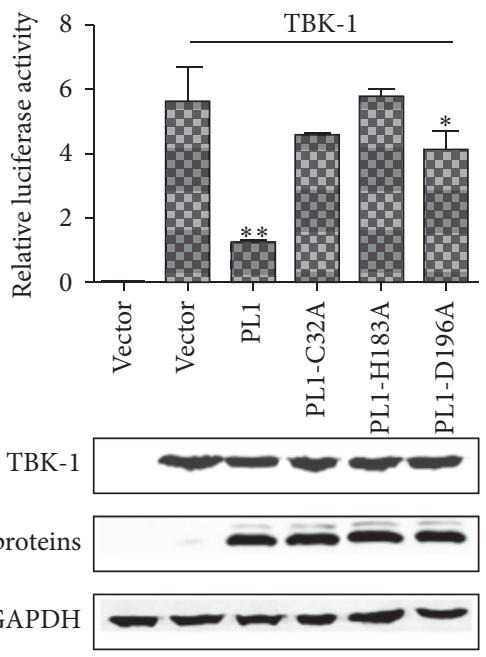

(e)

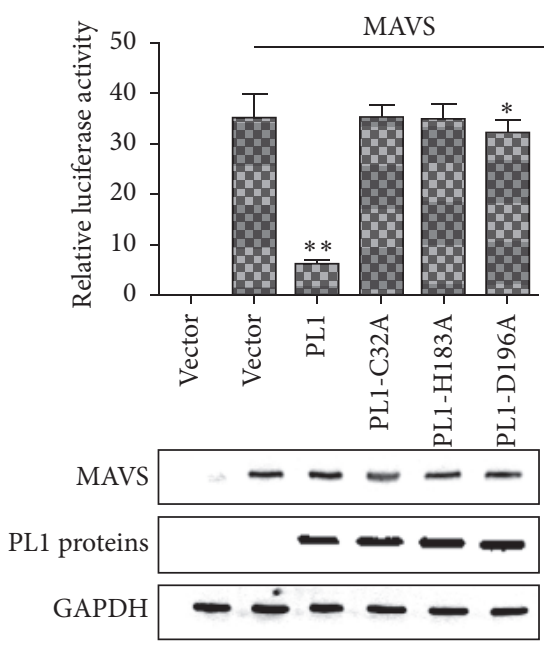

(c)



(f)

FIGURE 3: Effects of the TGEV PL1 catalytic mutants on expression of the IFN- $\beta$-Luc and localization of the protein. (a) HEK293T cells were cotransfected with the $500 \mathrm{ng}$ catalytic mutants C32A, H183A, and D196A, together with reporters of IFN- $\beta$-Luc and pRL-TK. Asterisks indicate statistical significance $(P<0.05)$. (b, c, d, e) HEK293T cells were transfected separately with RIG-1 (500 ng), MAVS (500 ng), STING (500 ng), or TBK-1 (500 ng), together with IFN- $\beta$-Luc and pRL-TK. Asterisks indicate statistical significance $(P<0.05)$. (f) Immunofluorescence microscopy of HeLa cells expressing Myc-TGEV PL1, Flag-STING, and DsRed-Mito. Cells were fixed 24 h after transfection, and Flag-tagged and Myc-tagged products were visualized using confocal microscopy.

activity (Figures 3(a), 3(b), 3(c), 3(d), and 3(e)). Based on the results, we conclude that the intact catalytic triad of TGEV PL1 is required to inhibit activation of the IFN- $\beta$ promoter driven by STING and TBK-1. Recent studies have revealed that STING acts as a scaffold protein for TBK-1 and IRF3 and links them to the MAVS complex in mitochondria upon viral infection [16]. Moreover, activation of STING is critical for stimulation of IRF-3 activity. Here, we observed that TGEV PL1 protein inhibits STING- and TBK-1- induced activation of IFN- $\beta$. Additional localization experiments showed that PL1 existed in mitochondria (Figure 3(f)).

3.4. TGEV PL1 Binds and Deubiquitinates RIG-I and STING. Modification of signaling molecules by ubiquitin (Ub) plays a critical role in activation of the IFN response. TGEV PL1 has been shown to possess DUB activity. Here, we investigated the DUB activities of TGEV PL1 and its catalytic mutants. HEK293T cells were cotransfected with pcDNA HA-Ub and TGEV PL1, and the level of ubiquitinated proteins was assessed via Western blot. The level of Ub-conjugated proteins was reduced dramatically in cells transfected with wild-type TGEV PL1, while the ubiquitinated Ub-HA level was not reduced in the presence of the C32A, H183A, and D196A mutants (Figure 4(a)). Next, we investigated whether TGEV PL1 recognizes and deubiquitinates the key regulators, RIG-I and STING, in the IFN signaling pathway. HEK293T cells were transfected with TGEV PL1, together with Flag-RIG-1 and Flag-STING for $24 \mathrm{~h}$, and cell lysates 




(a)

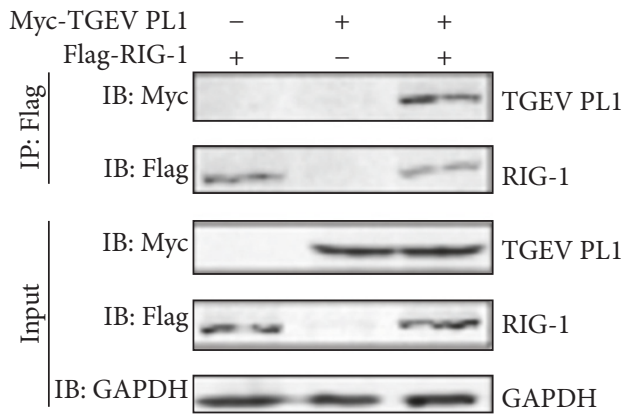

(b)



(c)

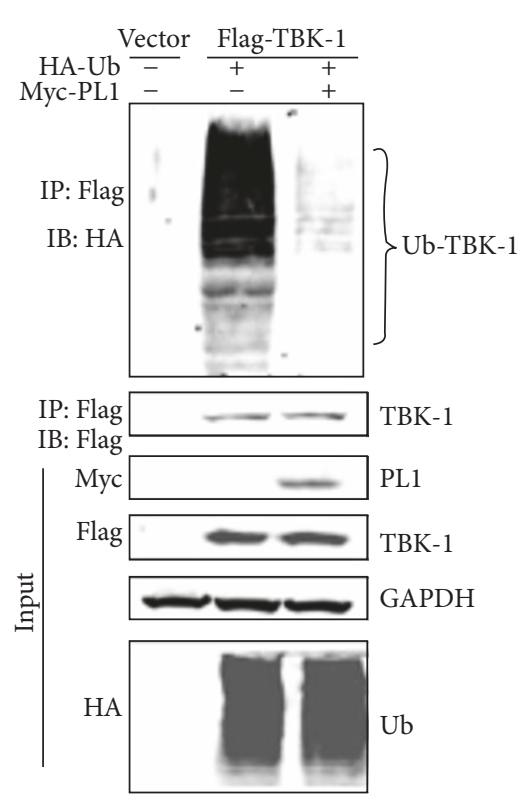

(d)

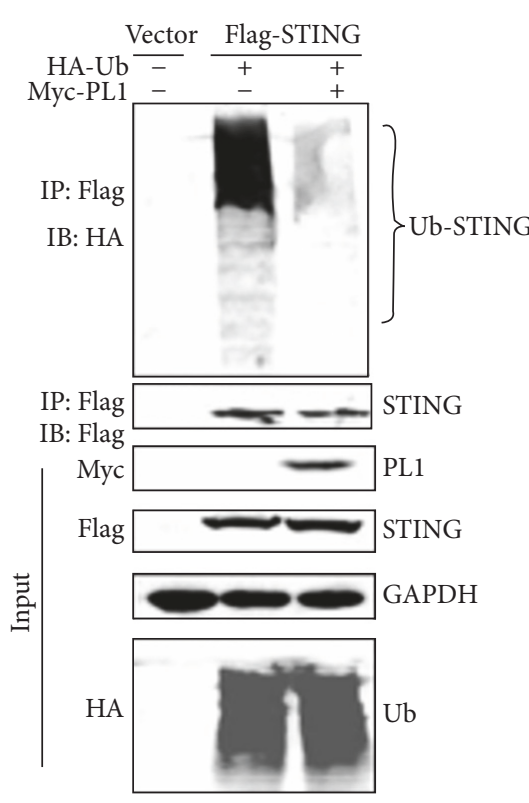

(e)

FIGURE 4: TGEV PL1 displays DUB activity that is dependent on its catalytic activity and reduces the ubiquitinated forms of RIG-I and STING. (a) HEK293T cells were transfected with HA-tagged ubiquitin and TGEV PL1 or the catalytic mutants C32A, H183A, and D196A. Proteins were assayed using Western blot with anti-HA and anti-Myc antibodies. ((b) and (c)) HEK293T cells were transfected with Myc-TGEVPL1 together with Flag-RIG-1 (b) and Flag-STING (c) for $24 \mathrm{~h}$, and lysates were subjected to coimmunoprecipitation and Western blot to determine the ubiquitination status of immunoprecipitated proteins. ((d) and (e)) HEK293T cells were transfected with Flag-RIG-1 (d) or Flag-STING (e), together with HA-Ub in the presence or absence of Myc-TGEVPL1. Cells were incubated for $24 \mathrm{~h}$ after transfection and then lysates were harvested. Lysates were immunoprecipitated with Flag, HA, and Myc antibodies and the products subjected to immunoblotting with anti-HA to evaluate ubiquitinated proteins.

were subjected to coimmunoprecipitation and Western blot to determine the ubiquitination of the immunoprecipitated protein. TGEV PL1 was detected in association with RIG-1 as well as STING (Figures 4(b) and 4(c), lane 3). Moreover, a dramatic reduction in the amount of ubiquitinated RIG-1 (Figure 4(d)) and STING (Figure 4(e)) was detected. These results suggest that TGEV PL1 antagonizes the IFN signaling pathway via deubiquitination of RIG-1 and STING.

\section{Discussion}

TGEV is known to induce robust expression of IFN- $\alpha$ at the late step of the replication and is distinct from CoVs
$[17,18]$. Moreover, TGEV infection activates transcription factors NF- $\kappa \mathrm{B}, \mathrm{IRF} 3$, and AP-1 in porcine kidney cells and a delayed activation of the IFN response in intestinal epithelial cells $[19,20]$. However, the mechanism of its evasion of the innate immune system has never been reported. The current study firstly showed antagonistic function of the TGEV PL1 protein against the IRF3 signaling pathway to inhibit IFN- $\beta$ induction through its DUB activity.

To combat the host antiviral effects, coronaviruses likely take advantage of PL activity to escape from the host innate antiviral response. HCoV-NL63 (PL2-TM) and SARS-CoV (PLpro-TM) inhibit STING-mediated activation of IRF-3 nuclear translocation and induction of IRF-3-dependent 
promoters $[6,8]$. PL2 of MHV strongly inhibits CARDIF-, TBK1-, and IRF3-mediated IFN- $\beta$ reporter activities and prevented nuclear translocation of IRF3 [7]. PEDV PLP2 negatively regulated RIG-I and STING-mediated IFN- $\beta$ expression [14]. Moreover, TGEV PL1 displays a similar structure to SARS-CoV PL2 [11] and gives rise to the speculation that TGEV PL1 may similarly act as an IFN antagonist. In the present study, we first found that overexpressed TGEV PL1 inhibited STING- and TBK-1-mediated IFN$\beta$ transcription and antagonized the type I IFN response stimulated by $\mathrm{SeV}$ in PK-15 cells. The catalytic activity of TGEV PL1 is essential for inhibiting IFN- $\beta$ transcription. Furthermore, STING dimerization is reduced in the presence of HCoV-NL63 PL2-TM, which was not affected by TGEV PL1. These results suggested that TGEV PL1 acted as an IFN antagonist to negatively regulate host antiviral innate immunity.

Ubiquitination and deubiquitination are critically involved in regulation of virus-induced type I IFN signaling pathways [21, 22]. Recently, DUBs have been reported in a variety of viruses, such as foot-and-mouth disease virus, Lpro [23], human cytomegalovirus, UL48 [24], herpes simplex virus type 1, UL36 [25], and porcine reproductive and respiratory syndrome virus, nsp2 [26, 27]. Interestingly, all CoVs have evolved to encode DUB enzymes, which may contribute to modulation of the innate immune response. PLP of HCoV-NL63, SARS-CoV, MHV, PEDV, and MERS$\mathrm{CoV}$ dramatically reduced the levels of ubiquitinated STING, RIG-I, TBK1, and IRF-3 to negatively regulate host antiviral innate immunity. Here, we showed that TGEV PL1 interferes with and significantly inhibits ubiquitination of RIG-1 and STING, which are essential activators of type I IFN signaling. Then, the levels of phosphorylated IRF-3 were reduced, which blocked nuclear translocation of IRF3 to activate the transcript of IFNs. Three catalytically inactive mutants of TGEV PL1 (C32A, H183A, and D196A) found to be defective in DUB activity failed to inhibit virus-induced INF- $\beta$ expression, indicating that the DUB function of TGEV PL1 is directly involved in inhibition of type I IFN induction. However, the membrane protein $\mathrm{M}$ and envelope protein $\mathrm{E}$ of TGEV were translated at the late step of the replication as the major inducing component of IFNs. Further studies are required to establish the precise functions of PL1 protease/DUB activity in coronavirus interactions with the host innate immune response.

\section{Conclusion}

Our results are the first report identifying TGEV PL1 that is responsible for inhibiting the induction of IFN- $\beta$. We found that TGEV PL1 displayed IFN antagonist activity dependent on the intact catalytic triad (C32, H183, and D196) and interfered with RIG-1- and STING-mediated signaling through a viral DUB activity. These characteristics of TGEV PL1 served as a multifunctional protein with a critical regulatory role in TGEV interactions with the host antiviral innate immune response. Moreover, these findings contribute to our understanding of the molecular mechanisms of innate immunity evasion strategies utilized by TGEV.

$\begin{array}{ll}\text { Abbreviations } \\ \text { CoVs: } & \text { Coronaviruses } \\ \text { HCoV-NL63: } & \text { Human coronavirus NL63 } \\ \text { SARS-CoV: } & \text { Severe acute respiratory syndrome CoV } \\ \text { MHV: } & \text { Murine hepatitis virus } \\ \text { PEDV: } & \text { Porcine epidemic diarrhea virus } \\ \text { MERS-CoV: } & \text { Middle East Respiratory Syndrome } \\ & \text { Coronavirus } \\ \text { PL: } & \text { Papain-like } \\ \text { SeV: } & \text { Sendai virus } \\ \text { IFN: } & \text { Interferon } \\ \text { TGEV: } & \text { Transmissible gastroenteritis virus } \\ \text { IRF3: } & \text { Interferon regulatory factor 3 } \\ \text { DUB: } & \text { Deubiquitinase } \\ \text { RIG-1: } & \text { Retinoic acid-induced gene I } \\ \text { STING: } & \text { Stimulator of interferon gene } \\ \text { PRR: } & \text { Pattern recognition receptors } \\ \text { PAMP: } & \text { Pathogen-associated molecular patterns } \\ \text { MDA5: } & \text { Melanoma differentiation gene 5 } \\ \text { IPS-1: } & \text { IFN- } \beta \text { promoter stimulator 1 } \\ \text { IKK: } & \text { I } \kappa \text { B kinase } \\ \text { TBK1: } & \text { TANK binding kinase 1 } \\ \text { NF- } \kappa \text { B: } & \text { Nuclear factor } \kappa \text { B } \\ \text { nsp5: } & \text { Nonstructural protein 5. } \\ & \end{array}$

\section{Conflicts of Interest}

The authors declare that they have no conflicts of interest regarding the publication of this paper.

\section{Acknowledgments}

This work was supported by the National Key Technology Support Program (2013BAK11B01-31), the state of international science and technology cooperation projects (2010DFB33620), and the National Natural Science Foundation of China (3140220).

\section{References}

[1] T. Kawai and S. Akira, "Antiviral signaling through pattern recognition receptors," The Journal of Biochemistry, vol. 141, no. 2, pp. 137-145, 2007.

[2] M. Yoneyama and T. Fujita, "RNA recognition and signal transduction by RIG-I-like receptors," Immunological Reviews, vol. 227, no. 1, pp. 54-65, 2009.

[3] H. J. Ramos and M. Gale Jr., "RIG-I like receptors and their signaling crosstalk in the regulation of antiviral immunity," Current Opinion in Virology, vol. 1, no. 3, pp. 167-176, 2011.

[4] L. Enjuanes, C. Smerdou, J. Castilla et al., "Development of protection against coronavirus induced diseases: A review," Advances in Experimental Medicine and Biology, vol. 380, pp. 197-211, 1995.

[5] J. Ziebuhr, E. J. Snijder, and A. E. Gorbalenya, "Virus-encoded proteinases and proteolytic processing in the Nidovirales," Journal of General Virology, vol. 81, no. 4, pp. 853-879, 2000.

[6] M. A. Clementz, Z. Chen, B. S. Banach et al., "Deubiquitinating and interferon antagonism activities of coronavirus papain-like 
proteases," Journal of Virology, vol. 84, no. 9, pp. 4619-4629, 2010.

[7] D. Zheng, G. Chen, B. Guo, G. Cheng, and H. Tang, "PLP2, a potent deubiquitinase from murine hepatitis virus, strongly inhibits cellular type I interferon production," Cell Research, vol. 18, no. 11, pp. 1105-1113, 2008.

[8] S. G. Devaraj, N. Wang, Z. Chen et al., "Regulation of IRF-3dependent innate immunity by the papain-like protease domain of the severe acute respiratory syndrome coronavirus," The Journal of Biological Chemistry, vol. 282, no. 44, pp. 3220832221, 2007.

[9] N. Barretto, D. Jukneliene, K. Ratia, Z. Chen, A. D. Mesecar, and S. C. Baker, "The papain-like protease of severe acute respiratory syndrome coronavirus has deubiquitinating activity," Journal of Virology, vol. 79, no. 24, pp. 15189-15198, 2005.

[10] A. M. Mielech, A. Kilianski, Y. M. Baez-Santos, A. D. Mesecar, and S. C. Baker, "MERS-CoV papain-like protease has deISGylating and deubiquitinating activities," Virology, vol. 450-451, pp. 64-70, 2014.

[11] J. A. Wojdyla, I. Manolaridis, P. B. Van Kasteren et al., "Papainlike protease 1 from transmissible gastroenteritis virus: Crystal structure and enzymatic activity toward viral and cellular substrates," Journal of Virology, vol. 84, no. 19, pp. 10063-10073, 2010.

[12] D. Wang, L. Fang, T. Li et al., "Molecular cloning and functional characterization of porcine IFN- $\beta$ promoter stimulator 1 (IPS1)," Veterinary Immunology and Immunopathology, vol. 125, no. 3-4, pp. 344-353, 2008.

[13] D. L. Vaux, F. Fidler, and G. Cumming, "Replicates and repeatswhat is the difference and is it significant? A brief discussion of statistics and experimental design," EMBO Reports, vol. 13, no. 4, pp. 291-296, 2012.

[14] Y. Xing, J. Chen, J. Tu et al., "The papain-like protease of porcine epidemic diarrhea virus negatively regulates type I interferon pathway by acting as a viral deubiquitinase," Journal of General Virology, vol. 94, no. 7, pp. 1554-1567, 2013.

[15] W. Sun, Y. Li, L. Chen et al., "ERIS, an endoplasmic reticulum IFN stimulator, activates innate immune signaling through dimerization," Proceedings of the National Acadamy of Sciences of the United States of America, vol. 106, no. 21, pp. 8653-8658, 2009.

[16] B. Zhong, Y. Yang, S. Li et al., "The adaptor protein MITA links virus-sensing receptors to IRF3 transcription factor activation," Immunity, vol. 29, no. 4, pp. 538-550, 2008.

[17] E. Albina, C. Carrat, and B. Charley, "Interferon- $\alpha$ response to swine arterivirus (PoAV), the porcine reproductive and respiratory syndrome virus," Journal of Interferon \& Cytokine Research, vol. 18, no. 7, pp. 485-490, 1998.

[18] P. Baudoux, C. Carrat, L. Besnardeau, B. Charley, and H. Laude, "Coronavirus pseudoparticles formed with recombinant $\mathrm{M}$ and E proteins induce alpha interferon synthesis by leukocytes," Journal of Virology, vol. 72, no. 11, pp. 8636-8643, 1998.

[19] Z. Ding, K. An, L. Xie et al., “Transmissible gastroenteritis virus infection induces NF- $\kappa \mathrm{B}$ activation through RLR-mediated signaling," Virology, vol. 507, pp. 170-178, 2017.

[20] L. Zhu, X. Yang, C. Mou, and Q. Yang, “Transmissible gastroenteritis virus does not suppress IFN- $\beta$ induction but is sensitive to IFN in IPEC-J2 cells," Veterinary Microbiology, vol. 199, pp. 128-134, 2017.

[21] V. G. Bhoj and Z. J. Chen, "Ubiquitylation in innate and adaptive immunity," Nature, vol. 458, no. 7237, pp. 430-437, 2009.
[22] M. K. Isaacson and H. L. Ploegh, "Ubiquitination, Ubiquitinlike Modifiers, and Deubiquitination in Viral Infection," Cell Host \& Microbe, vol. 5, no. 6, pp. 559-570, 2009.

[23] D. Wang, L. Fang, P. Li et al., "The leader proteinase of foot-andmouth disease virus negatively regulates the type I interferon pathway by acting as a viral deubiquitinase," Journal of Virology, vol. 85, no. 8, pp. 3758-3766, 2011.

[24] E. T. Kim, S. E. Oh, Y.-O. Lee, W. Gibson, and J.-H. Ahn, "Cleavage specificity of the UL48 deubiquitinating protease activity of human cytomegalovirus and the growth of an activesite mutant virus in cultured cells," Journal of Virology, vol. 83, no. 23, pp. 12046-12056, 2009.

[25] L. M. Kattenhorn, G. A. Korbel, B. M. Kessler, E. Spooner, and H. L. Ploegh, "A deubiquitinating enzyme encoded by HSV-1 belongs to a family of cysteine proteases that is conserved across the family Herpesviridae," Molecular Cell, vol. 19, no. 4, pp. 547557, 2005.

[26] Z. Chen, X. Zhou, J. K. Lunney et al., "Immunodominant epitopes in nsp2 of porcine reproductive and respiratory syndrome virus are dispensable for replication, but play an important role in modulation of the host immune response," Journal of General Virology, vol. 91, no. 4, pp. 1047-1057, 2010.

[27] Z. Sun, Z. Chen, S. R. Lawson, and Y. Fang, "The cysteine protease domain of porcine reproductive and respiratory syndrome virus nonstructural protein 2 possesses deubiquitinating and interferon antagonism functions," Journal of Virology, vol. 84, no. 15, pp. 7832-7846, 2010. 

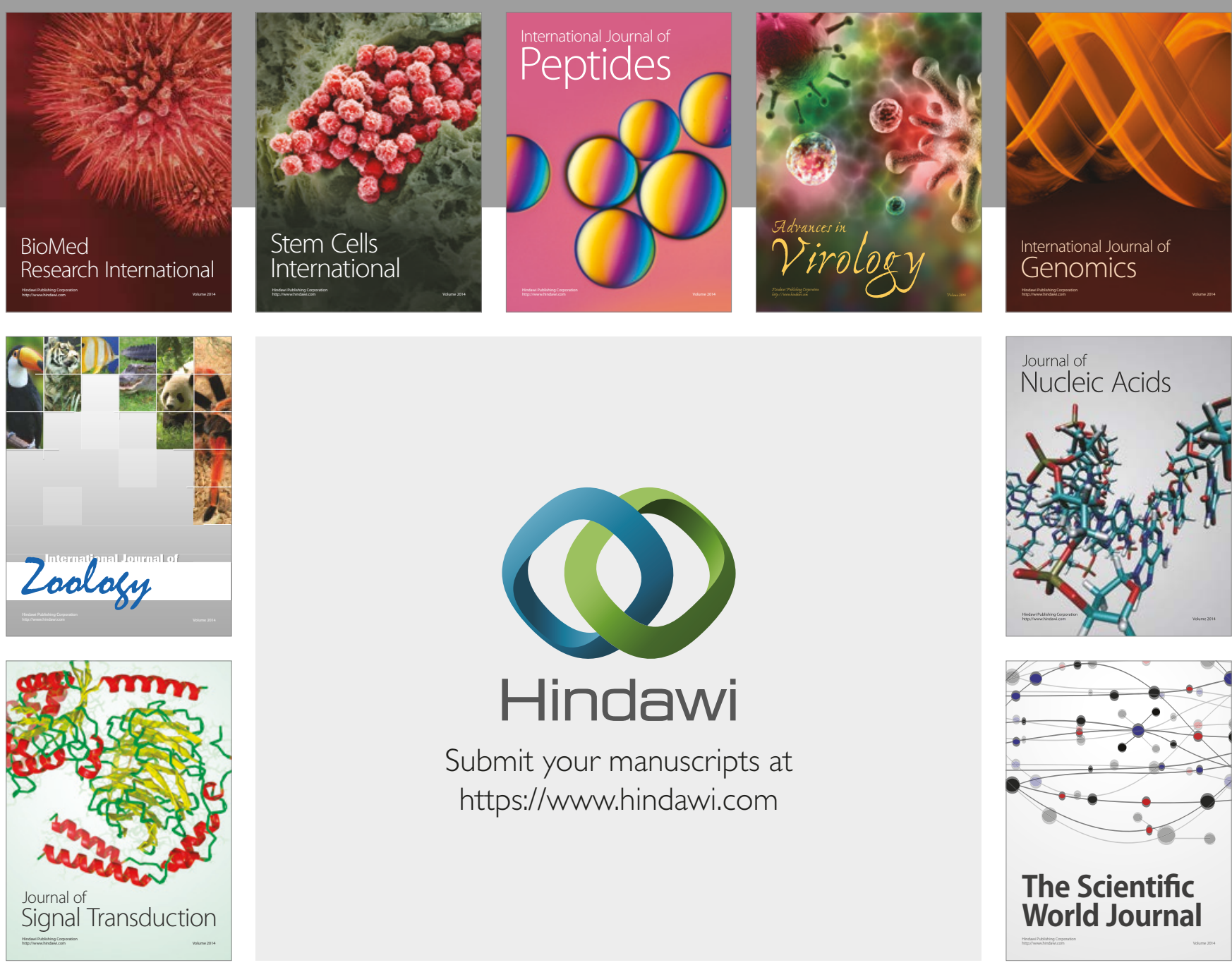

Submit your manuscripts at

https://www.hindawi.com
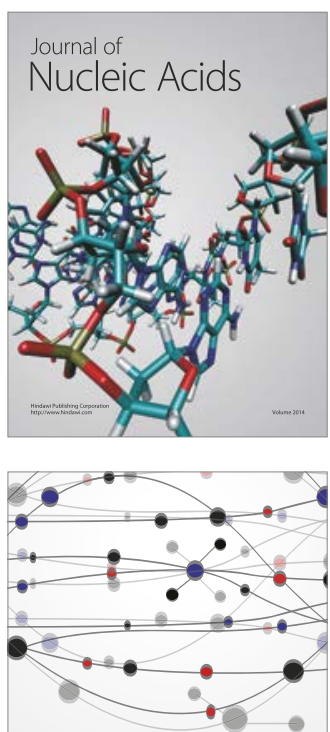

The Scientific World Journal

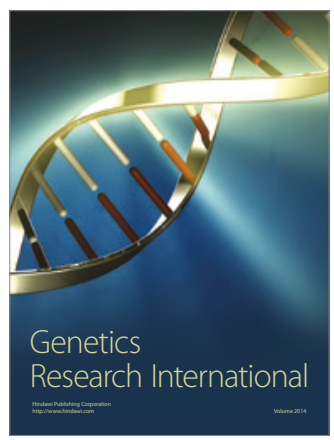



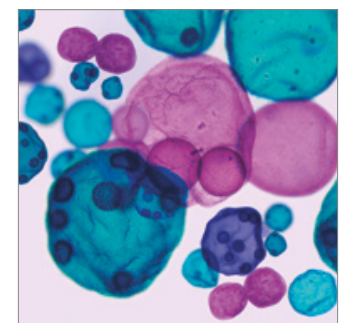

International Journal of Microbiology
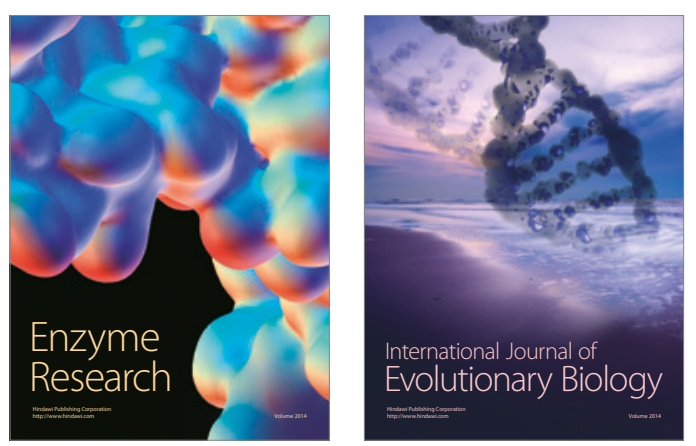
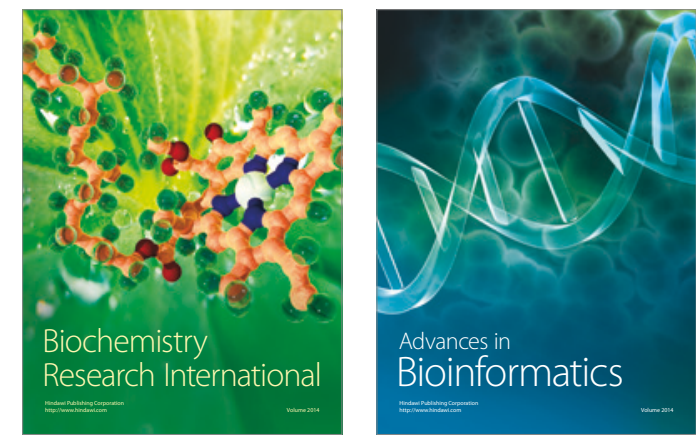

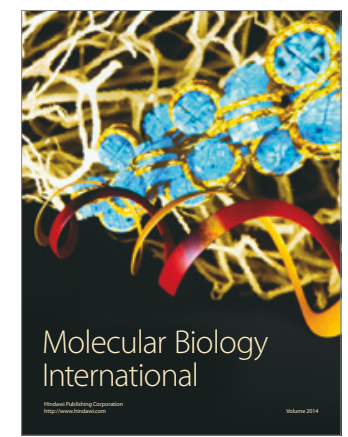

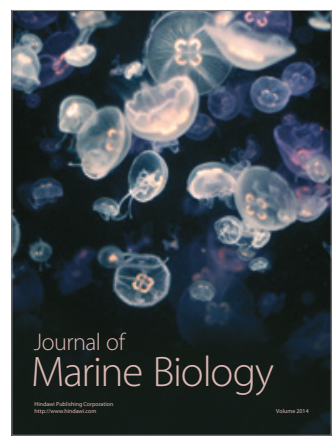

